\section{Scabies norvegica bei Diabetes mellitus}

Zusammenfassung. Die Skabies ist eine oft verkannte parasitäre Infektion, die sich häufig durch starken Juckreiz manifestiert. Infektionen mit sehr hoher Milbendichte werden als Scabies norvegica bezeichnet. Sie treten vorwiegend bei Patienten mit Immundefekten auf. Wir berichten über eine Patientin mit Diabetes mellitus, die an einer Scabies norvegica mit untypischem Verlauf erkrankte.

Scabies norvegica in Diabetes Mellitus. Scabies is an often mistaken parasitical infection, which usually shows strong pruritus. Norwegian scabies is characterised by the great number of parasites, and it occurs predominantly in patients with immunodeficiency. The following case report describes a patient with Diabetes mellitus Type I, suffering from Norwegian scabies with untypical development.

\section{Einleitung}

Die Skabies ist eine häufige, stark juckende, durch Acarus scabiei hominis hervorgerufene Dermatose. Als Ursache für den Pruritus wird eine allergische Reaktion auf Milbenbestandteile angesehen. Die Weibchen bohren Gänge in das Stratum corneum, wo sie ihre Eier ablegen. In drei bis vier Wochen entwickeln sich dort adulte Milben, und es kommt zur Reinfektion. Eine schlechte Immunabwehr und schlechte hygienische Lebensbedingungen können zu einer übermäßigen Ausbreitung der Milben führen; diese sogenannte Scabies norvegica ist eine seltene, hochkontagiöse Verlaufsform.

\section{Anamnese}

Eine 42-jährige Patientin wurde in der chirurgischen Klinik zur Behandlung ihrer Mala perforantes an beiden Füßen aufgenommen. Von dort kam es zur Verlegung in die Hautklinik zur Abklärung des bereits seit 2 Jahren bestehenden generalisierten Pruritus.

Akt Dermatol 2001; 27: 123-125

(c) Georg Thieme Verlag Stuttgart · New York ISSN 0340-2541
Birgit Mähnß, G. Itschert, K. Neuber

Universitätsklinik Hamburg-Eppendorf, Klinik für Dermatologie und Venerologie (Direktorin: Prof. Dr. I. Moll)

Anamnestisch bestanden seit 3 Jahren eine Onychomykose und eine Mykose der Zehenzwischenräume und Zehen, sowie vor etwa 2 Jahren eine Skabiesinfektion.

Während einer Gravidität vor 14 Jahren kam es zur Manifestation eines Diabetes mellitus Typ I.

\section{Dermatologischer Befund}

An den Füßen, mit Betonung der Fußsohlen, teils Hyperkeratosen, teils gelbliche Schuppen und Schuppenkrusten (Abb.1). Plantar ödematöse Schwellung und Fistelgänge, aus denen sich Eiter entleerte.

Über das gesamte Integument außer interdigital, paraumbilikal, axillär und inguinal fanden sich Papeln und Exkoriationen neben kreisrunden, z.T. narbigen Hyperpigmentierungen (Abb. 2).

\section{Laborbefunde}

Außer einem erhöhten Glukosespiegel im Serum (220 mg/dl) und einem HbA1c von 14,6\% sowie einem CRP von $22 \mathrm{mg} / \mathrm{dl}$ ( $\mathrm{NB}<10 \mathrm{mg} / \mathrm{dl}$ ) keine pathologischen Blutbefunde. Im Kalilaugen-Präparat (Hautschuppen Füße) fanden sich massenhaft Skabiesmilben, -eier und Kot (Abb. 3).

\section{Therapie und Verlauf}

Die Scabies-norvegica-artige Infektion mit Acarus scabiei wurde zunächst für 3 Tage am gesamten Integument mit Jacutin ${ }^{\circledR}$ Emulsion behandelt. Aufgrund der starken Schwellungen an den Füßen waren die dortigen Fistelgänge der Therapie schlecht zugänglich; deshalb wurde eine einmalige Gabe von $1 \times 12 \mathrm{mg}$ p.o. Ivermectin angeschlossen. Durch den darauffolgenden Einsatz von lokalen Steroiden kam es zur raschen Befundbesserung und Rückbildung des Juckreizes.

Weiterhin wurden die Fistelgänge täglich mit Lavasept-Lösung gespült und die Füße in Kaliumpermanganatlösung gebadet. Wegen des erhöhten CRP-Wertes und einer Osteolyse des Digitus III am rechten Fuß zusätzlich Antibiose über 14 Tage mit $3 \times 600 \mathrm{mg}$ Sobelin ${ }^{\circledR}$ i.v. Bei Entlassung war das CRP normwertig und die Fistelgänge waren frei von Eiter. 


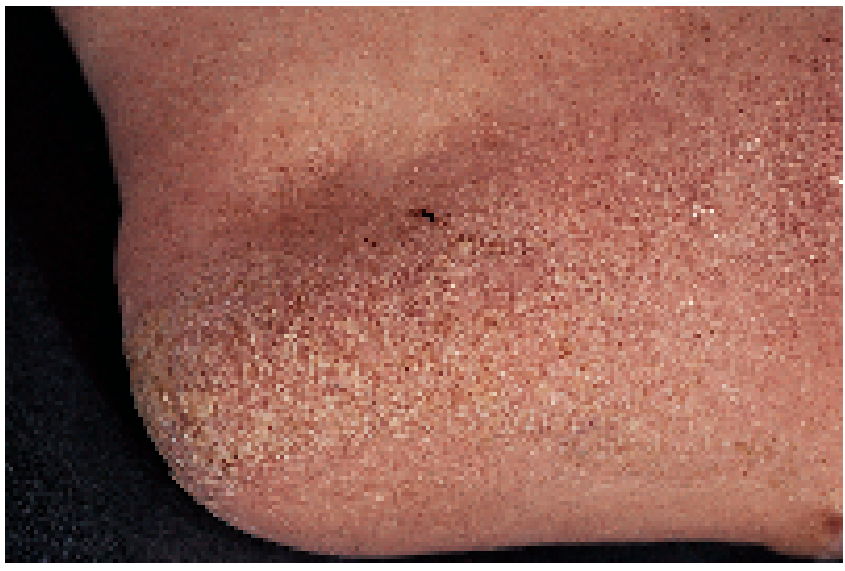

Abb.1 Fußrand mit hyperkeratotischen, stark schuppenden Plaques.

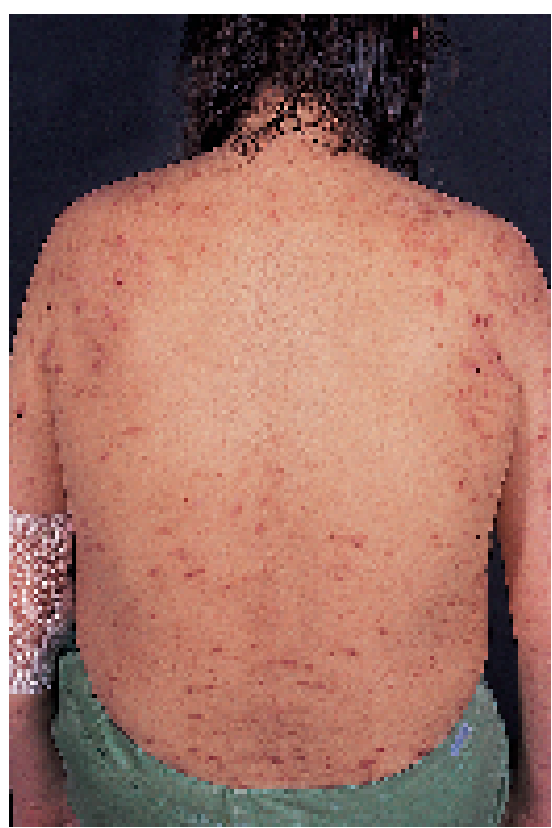

Abb. 2 Papeln und Exkoriationen am Integument.

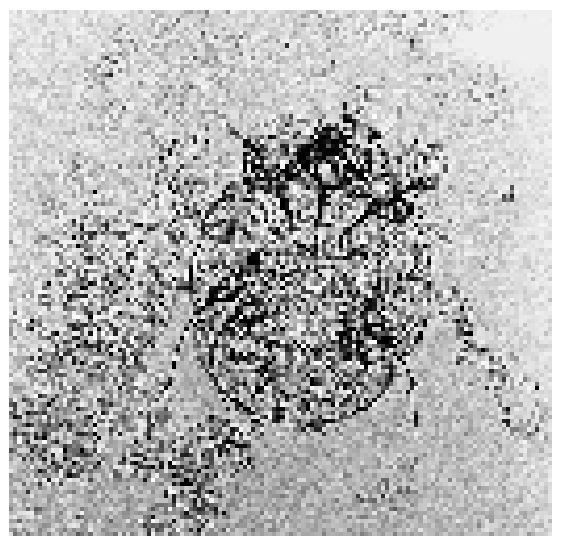

Abb. 3 Scabiesmilbe im Kalilaugenpräparat $(\times 320)$.

\section{Diskussion}

Obwohl bei der Patientin eine 2 Jahre zurückliegende Skabiesinfektion bekannt war, wurde initial nicht an ein Rezidiv gedacht, vor allem deshalb, weil die üblichen Prädilektionsstellen (interdigital, paraumbilikal, axillär) ausgespart waren. Der dermatologische Befund am Körperstamm zeigte die klinischen Symptome einer Prurigo simplex subacuta, die Hyperkeratosen der Fußsohlen ließen primär an eine Mykose denken.

Allerdings findet man in der Literatur durchaus Hinweise auf ungewöhnliche klinische Befunde bei der Scabies norvegica $[5,6]$.

Diese Sonderform der Skabies ist gekennzeichnet durch das massive Auftreten von Milben und ist deshalb extrem kontagiös. Sie wird hauptsächlich bei debilen Patienten, z. B. bei seniler Demenz, sensorischer Neuropathie oder nicht immunkompetenten Patienten beobachtet.

Neben Kindern und alten Menschen sind deshalb auch immunsupprimierte Patienten besonders betroffen. Die Immunsuppression kann sowohl durch eine entsprechende Erkrankung (z.B. AIDS), als auch durch entsprechende Therapien bedingt sein, z. B. nach Knochenmarkstransplantation [1,2].

In letzter Zeit wurden auch Fälle von Scabies norvegica nach längerer Applikation von topischen Kortikosteroiden beschrieben [3].

Bei unserer Patientin lag seit über 10 Jahren ein Diabetes mellitus vor, der schlecht eingestellt war. Die Folge war eine Reihe von sekundären Komplikationen. Im Vordergrund standen Mala perforantes aufgrund der diabetischen Mikroangiopathie und der diabetischen Polyneuropathie.

Hinweise auf eine erhöhte Empfindlichkeit gegenüber Infekten sowie die Mechanismen der Immundefizienz bei Diabetes mellitus sind beschrieben [4]. Danach ist die Immunkompetenz aller Leukozytenpopulationen verringert, wobei die Funktionen der polymorphkernigen neutrophilen Leukozyten eine deutliche Verschlechterung der Chemotaxisfunktionen zeigen. Aber auch indirekte Effekte des Insulinmangels, z. B. die Hyperglykämie, können in vitro eine Verschlechterung der Abwehrfunktionen gegen verschiedene Erreger bewirken.

Einen Zusammenhang zwischen Diabetes mellitus und Scabies norvegica findet man in der Literatur hingegen nicht. Nach unseren Beobachtungen besteht bei älteren Patienten mit Skabiesinfektion häufig zusätzlich ein insulinpflichtiger Diabetes mellitus. Eine gestörte Hautbarriere und auch die gestörte Immunität könnten dabei eine pathophysiologische Rolle spielen.

Dieser Fall von Scabies norvegica zeigt, dass bei Diabetes mellitus und generalisiertem Juckreiz auch an eine Skabiesinfektion gedacht werden muss, selbst wenn Klinik und Anamnese nicht typisch sind. 


\section{Literatur}

${ }^{1}$ Barnes L, Mac Callister RE, Lucky AW. Crusted (Norwegian) scabies. Occurrence in a child undergoing a bone marrow transplant. Arch Dermatol 1987; 123: 95-97

2 Del Giudice P, Charles M, Couppie P, Bernard E, Lacour JP, Marty P, et al. Successful treatment of crusted (Norwegian) scabies with ivermectin in two patients with human immunodeficiency virus infection. Br J Dermatol 1996; 135: 494-495

${ }^{3}$ Marlière V, Roul S, Labreze C, Taieb A. Crusted (Norwegian) scabies induced by use of topical corticosteroids and treated successfully with ivermectin. J Pediatr 1999; 135: 122 - 124

${ }^{4}$ Moutschen MP, Scheen AJ, Lefebvre PJ. Impaired immune responses in diabetes mellitus: analysis of the factors and mechanisms involved. Relevance to the increased susceptibility of diabetic patients to specific infections. Diabete Metabolism 1992; 18: 187 201

${ }^{5}$ Nowak MA, Mirando WS, Brodell RT. Crusted (Norwegian) scabies. A simple office test demystifies the „great imitator“. Postgrad Med 1999; 105: $153-156$

${ }^{6}$ Trefzer D, Vanscheidt W. Scabies. Fortschr Med 1999; 117: 57 - 58

Prof. Dr. Karsten Neuber

Klinik für Dermatologie

Martinistraße 52

20246 Hamburg

E-mail: neuber@uke.uni-hamburg.de

\section{BUCHBESPRECHUNG}

Braun-Falco, O., G. Plewig, H. H. Wolff, W. H. C. Burgdorf: Dermatology. 2nd rev. ed. Berlin, Heidelberg, New York: Springer 2000. 1853 S., 1038 Farbabb., 281 Tab. Geb. DM 398,-. ISBN 3-540-59452-3

Das vorliegende Standardlehrbuch der deutschsprachigen Dermatologie steht nunmehr in seiner zweiten, komplett überarbeiteten, englischsprachigen Ausgabe zur Verfügung. Es ist den Autoren sehr gut gelungen, in 72 Kapiteln in einem umfangreichen Band die gesamte Dermatologie einschließlich ihrer Grenzgebiete kompetent darzustellen. Zur Komplettierung des Gesamtwerkes wurden zwei neue Kapitel über Dermatosen in pigmentierter Haut und das der operativen Dermatologie hinzugefügt. Insgesamt besticht das Werk durch seine klare Gliederung, gute tabellarische Aufarbeitung und hervorragende Farbfotografien sowie eine bei den meisten Beiträgen aktualisierte Literaturübersicht. Der umfassende Wissenszuwachs der letzten Jahre wurde in relevanter Form in diese zweite überarbeitete Auflage integriert, ohne den Gesamtrahmen zu sprengen. Somit stellt dieses Lehrbuch ein Standardwerk der modernen Dermatologie dar sowie ein informatives Nachschlagewerk für jeden klinisch tätigen Dermatologen wie Nicht-Dermatologen. W. H. C. Burgdorf ist mit dieser zweiten, komplett überarbeiteten Ausgabe eine hervorragende englischsprachige Übersetzung gelungen. Hierdurch wird dem primär deutschsprachigen traditionsreichen Lehrbuch O. Braun-Falco, G. Plewig und H. H. Wolff eine internationale Veröffentlichung und Leserschaft ermöglicht. Die Herausgeber haben es exzellent verstanden, die bei einem so großen Werk notwendigen Mitautoren homogen in das Gesamtkonzept, das diesem Buch zugrunde liegt, einzubinden. Als Standardwerk gehört dieses Lehrbuch zur Basisausstattung eines jeden klinischen tätigen Dermatologen und Nicht-Dermatologen wie auch interessierten Studenten.

R. Stadler, Minden 